\title{
Síndrome de Cowden: presentación de un caso clínico con lesiones orales
}

\author{
R. ALMENAR BESÓ, J. VICENTE BAGÁN SEBASTIÁN, Mª . A. MILIÁN MASANET, \\ Y. JIMÉNEZ SORIANO
}

Servicio de Estomatología. Hospital General Universitario. Valencia

COWDEN SYNDROME: A CLINICAL CASE WITH ORAL LESIONS

\begin{abstract}
RESUMEN
El síndrome de Cowden es una enfermedad hereditaria, de transmisión autosómica dominante, caracterizada por la presencia de múltiples hamartomas y nódulos en piel y mucosa oral, junto con anomalías en mama, tiroides y pólipos en el tracto gastrointestinal, tendiendo a malignizarse sobre todo en mama y tiroides. El diagnóstico precoz a partir de las lesiones orales puede dar lugar a que se descubran otras tumoraciones, asintomáticas, en otras partes del organismo. Así ocurrió en el caso clínico que a continuación expondremos, en el que a la paciente, tras la sospecha de un síndrome de Cowden por la presencia de múltiples fibromas en la mucosa oral, se le diagnosticó un carcinoma de mama, y otras alteraciones como calcificaciones a nivel tiroideo y pólipos intestinales.
\end{abstract}

PALABRAS CLAVE:Síndrome de Cowden. Hamartomas múltiples. Oral.

\begin{abstract}
Cowden syndrome is an autosomal-dominant inheritance disease, characterized by the presence of skin and oral mucosa multiple hamartomas and nodules, together with thyroid and breast anomalies and polyposis of the gastrointestinal tract, which tend to undergo malignant transformation, especially in breast and thyroids. Therefore, the oral lesions early diagnosis facilitates the identification of asintomatic lesions, in other parts of the body. This is the case in the report we are to present, in which the patient, probably with the Cowden Syndrome, because of the presence of multiple hamartomas in the oral mucose diag nosed a breast carcinoma, and other alterations such as thyroid calci fications and polyposis of the gastrointestinal tract.
\end{abstract}

KEY WORDS: Cowden Syndrome. Multiple hamartomas. Oral.

Almenar Besó R, Vicente Bagán Sebastián J, Milián Masanet MaA, Jiménez Soriano Y. Síndrome de Cowden: presentación de un caso clínico con lesiones orales. An Med Interna (Madrid) 2001; 18: 426-428.

\section{INTRODUCCIÓN}

El síndrome de Cowden, también llamado síndrome de hamartomas múltiples o síndrome neoplásico (1-5), es una enfermedad hereditaria, de transmisión autosómica dominante con penetrancia incompleta y de expresividad variable $(6,7)$. Fue descrita por Lloyd y Dennis en 1963, denominándola con el nombre de su paciente.

Se caracteriza por la presencia de hamartomas múltiples procedentes de las tres hojas blastodérmicas, con alto riesgo de malignización $(2,5,8)$.

Clínicamente observamos hamartomas y nódulos en piel y mucosa oral, junto con anomalías en mama, tiroides y pólipos en el tracto gastrointestinal. Estas lesiones cutaneomucosas son el principal criterio diagnóstico (3) y tiene una gran importancia en Odontología, ya que la identificación de lesiones orales facilita el diagnóstico temprano de otras alteraciones en órganos y sistemas $(1,2,4,5,9,10)$.
El caso clínico que a continuación presentamos se trata de una paciente que tenía múltiples fibromas en la mucosa oral, en base a los cuales, tras pensar que se podría tratar de un síndrome de Cowden, se comprobó que también existían lesiones en la mama y tiroides, en el primer caso con caracteres de malignidad.

\section{CASO APORTADO}

Mujer, de 71 años de edad, que acudió a nuestro Servicio de Estomatología del Hospital General de Valencia, por presentar múltiples lesiones de aspecto fibromatoso, de 1-2 mm cada una, en reborde alveolar edéntulo superior e inferior (Fig. 1) y en dorso de lengua, de varios años de evolución, sin poder precisarlo exactamente. Las lesiones eran asintomáticas, pero le impedían colocarse la prótesis.

Se realizó una biopsia de la lesión del reborde alveolar inferior, informándonos de un epitelio paraqueratósico y acantósico con pre-

Trabajo aceptado: 10 de Enero de 2000

Correspondencia: R. Almenar Besó. Servicio de Estomatología. Hospital General Universitario de Valencia. Avda. Blasco Ibáñez,17. 46014 Valencia. 


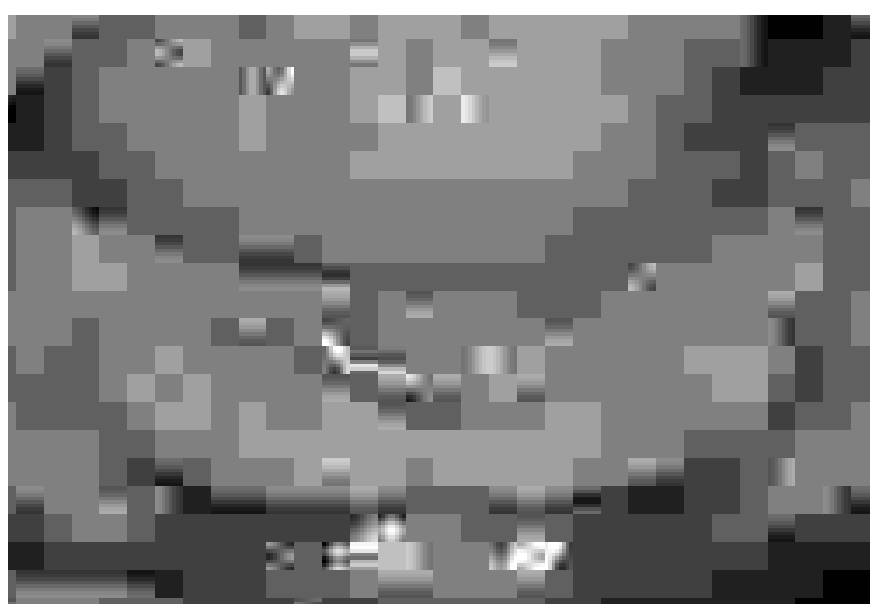

Fig. 1. Lesiones de aspecto fibromatoso en reborde edéntulo.

sencia de microabscesos de polimorfonucleares en la capa córnea, dando el diagnóstico de hiperplasia epitelial benigna (Fig. 2).

La paciente presentaba máculas cutáneas de gran tamaño hipomelánicas, de varios años de evolución, cuyo diagnóstico fue de vitíligo peribucal,en antebrazo a nivel de codos y pies.

Simultáneamente, para descartar un posible síndrome de Cowden, se realizaron distintas pruebas complementarias, tales como radiografía de tórax, ecografía de mama y tiroides,tránsito intestinal y estudio ginecológico.

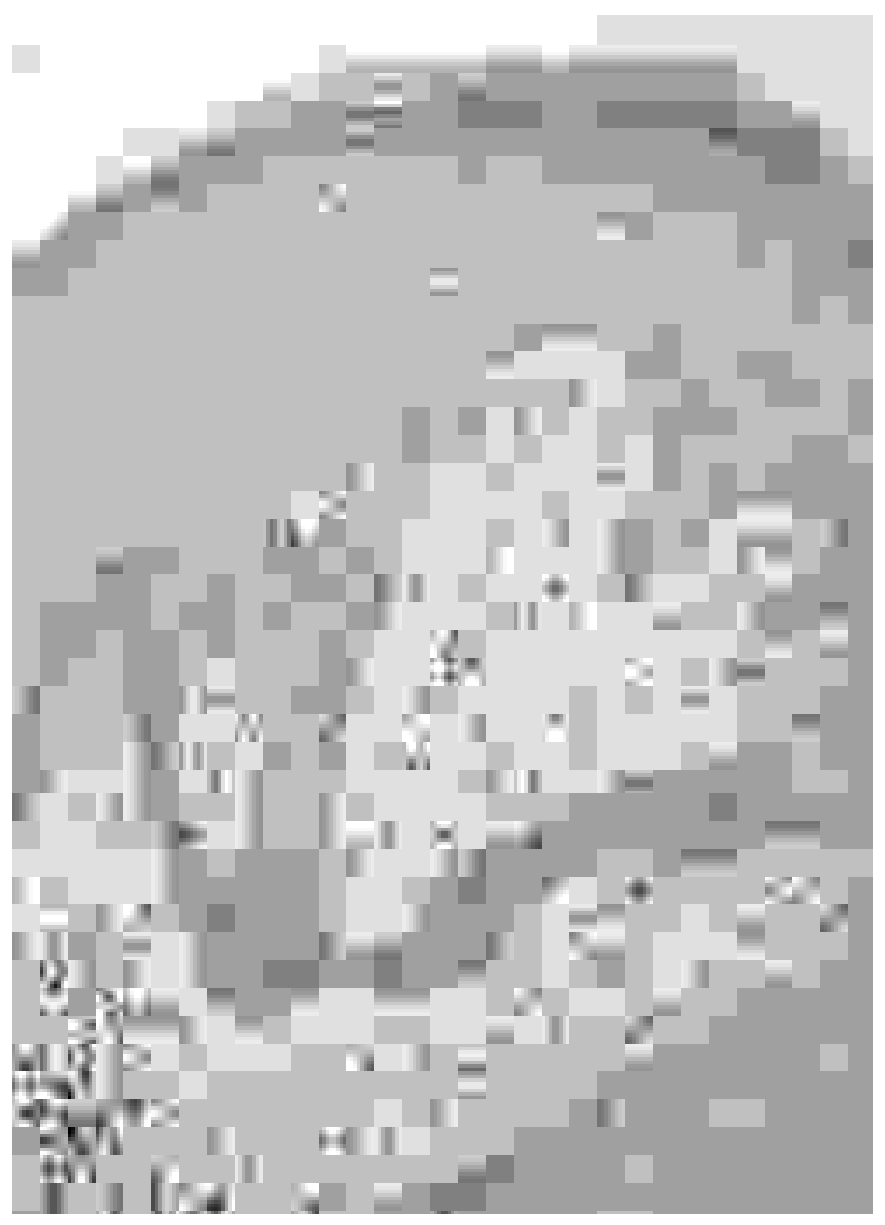

Fig. 2. Epitelio paraqueratósico y acantósico con presencia de microabscesos de polimorfonuclares en la capa córnea.
La paciente presentaba múltiples pólipos intestinales. En el lóbulo tiroideo derecho se observaron varias calcificaciones, situadas sobre todo a nivel del tercio inferior. El lóbulo tiroideo izquierdo presentaba un nódulo sólido de $1,2 \mathrm{~cm}$ y a nivel del tercio inferior otro nódulo más pequeño de $0,7 \mathrm{~cm}$.

También se encontraron en la ecografía mamaria múltiples fibroadenomas calcificados dispersos por ambas mamas, al igual que varios quistes mamarios. En la mama izquierda se observaba una neoplasia de $2,5 \mathrm{~cm}$. En la biopsia mamaria se confirmó el diagnóstico de carcinoma ductal infiltrante de mama. A la vez se realizó una mastectomía y linfadenectomía axilar. Se le pidió una tomografía axial computerizada (TAC ) torácica, la cual nos informaba que no existían adenopatías mediastínicas, pero si una metástasis costal. El TAC del abdomen nos señaló una discreta hepatomegalia, sin embargo la ecografía nos mostraba un hígado de aspecto heterogéneo con porta vesícula y colédoco normales. El bazo, páncreas, suprarrenal derecha y ambos riñones estaban dentro de la normalidad, salvo pequeños quistes parapiélicos en el riñón derecho y corticales. En la glándula suprarrenal izquierda se observaba un nódulo hipodenso. La paciente dada su enfermedad metastásica recibió un tratamiento quimioterápico con ciclofosfamida, metotexate, 5-fluoracilo $(\mathrm{CMF})$. Actualmente habiendo transcurrido un año tras el diagnóstico de su enfermedad se encuentra con metástasis óseas y hepáticas.

\section{DISCUSIÓN}

El síndrome de Cowden presenta un ligero predominio en el sexo femenino $(60 \%)$, y afecta preferentemente a la raza blanca $(96 \%)(1,6)$. Nuestro caso clínico coincide con dichas características. En cuanto a la edad, suele manifestarse antes de la tercera década de la vida (2).

La presencia de pápulas faciales cutáneas y la papilomatosis de la mucosa oral son los criterios clínicos mayores para establecer el diagnóstico del síndrome de Cowden $(4,6)$, debiendo alertar al clínico ante la posibilidad de otras lesiones neoplásicas, a veces ocultas, sobre todo en mama y tiroides con carácter maligno $(1,2,6,11,12)$. Así pudo comprobarse en nuestro caso donde la paciente presentaba un carcinoma en mama, que era asintomático sin tener conocimiento la paciente de él y siendo diagnosticado a partir de los signos orales.

El síndrome de Cowden se caracteriza por la presencia de múltiples hamartomas, los cuales se pueden manifestar intraoralmente como pequeñas pápulas, papilomatosis y formaciones verrugosas. La mayoría de los pacientes (más del 80\%) presentan pápulas o lesiones verrugosas en labios, lengua, encía, reborde alveolar edéntulo, mucosa yugal (3), paladar, úvula y ocasionalmente en el istmo de las fauces o la orofaringe (6). Las prótesis mal adaptadas pueden contribuir a aumentar estas lesiones con su irritación (1). Las lesiones son fibromas y no presentan un cuadro histológico característico. Nuestro paciente tenía múltiples fibromas localizados en el reborde alveolar edéntulo superior e inferior, y en lengua.

Dermatológicamente pueden aparecer pápulas liquenoides en la piel facial, asociados a múltiples tricholemomas $(2,8)$, así como queratosis palmoplantar (2). En cambio nuestro paciente presentaba vitíligo en piel.

En mama, puede manifestarse como una enfermedad fibroquística y papilomas ductales en el $76 \%$ de los casos $(1,6)$, también como fibradenomas o adenocarcinomas (3). Nuestro paciente presentaba múltiples fibroadenomas calcificados, quistes mamarios en ambas mamas y una neoplasia de mama izquierda (carcinoma ductal infiltrante). 
En cuanto a la patología tiroidea se presenta como bocios multinodulares en un $67 \%(1,3,6)$, coincidiendo con nuestro caso, el cual tenía varias calcificaciones y nódulos tiroideos. Los casos presentados por Mignogna y cols. (11), se trataban de pacientes que tenían adenomas en las glándulas tiroideas, por lo que se les realizó una tiroidectomía, sin embargo no presentaban lesiones en mama, aunque no por ello hay que olvidarse de revisar a estos pacientes periódicamente y realizarles mamografías bilaterales entre 6 y 12 meses.

Como patología del tracto digestivo, encontramos pólipos de esófago y gastrointestinales (6). En nuestro caso la paciente presentaba múltiples pólipos intestinales. En nuestro articulo previo (1) ya señalamos que se presentan en los pacientes múltiples formaciones papilomatosas en esófago, estómago, duodeno, colon y recto.

\section{Bibliografía}

1. Bagán JV, Peñarrocha M, Vera-Sempere F. Cowden syndrome: Clinica and pathological considerations in two new cases. J Oral Maxillofac Surg 1989; 47: 291-4.

2. Thomas DW, Lewis MAO. Lhermitte-Duclos disease associated with Cowden's disease. Int J Oral Maxillofac Surg 1995; 24: 369-71.

3. Porter S, Cawson R, Scully C, et al. Multiple hamartoma syndrome presenting with oral lesions. Oral Surg Oral Med Oral Pathol Oral Radiol Endod 1996; 82: 295-301.

4. Takenoshita Y, Kubo S, Takeuchi $\mathrm{T}$ et al. Oral and facial lesions in Cowden's disease:Report of two cases and a review of the literature. J Oral Maxillofac Surg 1993; 51: 682-7.

5. Swart JG, Lekkas C, Allard RH. Oral manifestations in Cowden's syndrome. Report of four cases. Oral Surg Oral Med Oral Pathol 1985; 59: 264-8.

6. Seoane J, Aguado A, Vázquez J et al. Síndrome de Cowden: manifesta -
El síndrome de Cowden puede asociarse a otras muchas enfermedades, así en el aparato genital, con quistes de ovario; a la enfermedad de Lhermitte-Duclos, meningiomas, hemangiomas, neurofibromas, linfoma no Hodgkin $(2,3)$, etc, también a quistes, hipoplasia de paladar blando y úvula, microstomía, hipoplasia mandibular y maxilar y maloclusión dental, policaries, pérdida de dientes (1), lengua escrotal, acortamiento radicular, manchas café con leche, así como anomalías esqueléticas del tipo macrocefalia, fascies adenoide, paladar arqueado, cifoscoliosis, y pectus excavatum (6).

En conclusión, debemos resaltar la importancia de las lesiones orales en este síndrome que pueden ayudarnos a sospechar y así poder diagnosticar neoplasias ocultas en otras partes del organismo.

ciones orales. Estudio clínico-patológico de un caso. Quintessence (ed esp) 1996; 9: 462-5.

7. Krasovec M, Elsner P, Burg G. Cowden's disease. Hautarzt 1995; 46 : 472-6.

8. Cohen M. Perspectives on craniofacial asymmetry. VI. The hamartoses. Int J Oral Maxillofac Surg 1995; 24: 195-200.

9. Kuffer R, Rougier M, Laugier P et al. Cowden's disease. A report on two cases in Swiss families. Rev Stomatol Chir Maxillofac 1979; 80: 246-56.

10. Mallory SB. Cowden syndrome (multiple hamartoma syndrome). Dermatol Clin 1995; 13: 27-31

11. Mignogna MD, Lorenzo MS ${ }^{\mathrm{a}}$, Ruocco V et al. Early diagnosis of multiple hamartoma and neoplasia syndrome (Cowden disease). The role of the dentist . Oral Surg Oral Med Oral Pathol Oral Radiol Endod 1995; 79: 295-9.

12. Gilbert HD, Plezia RA, Pietruk T. Cowden's disease (multiple hamartoma syndrome). J Oral Maxillofac Surg 1985; 43: 457-60. 